\title{
A 2-D Orientation-Adaptive Prediction Filter in Lifting Structures for Image Coding
}

\author{
Ömer N. Gerek, Member, IEEE, and A. Enis Çetin, Member, IEEE
}

\begin{abstract}
Lifting-style implementations of wavelets are widely used in image coders. A two-dimensional (2-D) edge adaptive lifting structure, which is similar to Daubechies $5 / 3$ wavelet, is presented. The 2-D prediction filter predicts the value of the next polyphase component according to an edge orientation estimator of the image. Consequently, the prediction domain is allowed to rotate $\pm 45^{\circ}$ in regions with diagonal gradient. The gradient estimator is computationally inexpensive with additional costs of only six subtractions per lifting instruction, and no multiplications are required.
\end{abstract}

Index Terms-Adaptive wavelet transform, image coding, orientation adaptive lifting.

\section{NOTATION}

W E introduce here the notations used throughout the paper. In order to illustrate the image operations, we consider a $3 \times 3$ portion of the image, centered around the center pixel $x[m, 2 n]$ as shown in Fig. 1 . The polyphase decomposition is explained along horizontal direction. Along this direction, the dashed pixels belong to polyphase component 1 , others belong to polyphase component 2 . We first define four gradient approximations around $x[m, 2 n]$ along angles of $135^{\circ}, 0^{\circ}, 45^{\circ}$, and $180^{\circ}$ with the horizontal axis:

- $\Delta_{135}=|x[m-1,2 n-1]-x[m+1,2 n+1]|$;

- $\Delta_{0}=|x[m, 2 n-1]-x[m, 2 n+1]| ;$

- $\Delta_{45}=|x[m+1,2 n-1]-x[m-1,2 n+1]| ;$

- $\Delta_{180}=|x[m-1,2 n]-x[m+1,2 n]|$.

Next, we define four possible prediction values for $x[m, 2 n]$ using its eight neighbors:

- $\hat{x}_{135}[m, 2 n]=(x[m-1,2 n-1]+x[m+1,2 n+1]) / 2$;

- $\hat{x}_{0}[m, 2 n]=(x[m, 2 n-1]+x[m, 2 n+1]) / 2$;

- $\hat{x}_{45}[m, 2 n]=(x[m+1,2 n-1]+x[m-1,2 n+1]) / 2$;

- $\hat{x}_{180}[m, 2 n]=(x[m+1,2 n]+x[m-1,2 n]) / 2$.

Among these gradient approximations and predictions, only $\Delta_{180}$ and $\hat{x}_{180}$ cannot be obtained from polyphase component 1.

We use $h_{0}$ as the low-pass analysis filter and $h_{1}$ as the highpass analysis filter in a subband decomposition structure. Con-

Manuscript received June 24, 2004; revised March 29, 2005. This work was supported in part by the Anadolu University Research Fund under Contract 030263, in part by a TUBITAK-TOGTAG-NSF grant, and in part by EU FP6 NoE: MUSCLE. The associate editor coordinating the review of this manuscript and approving it for publication was Prof. Trac. D. Tran.

O. N. Gerek is with the Department of Electrical and Electronics Engineering, Anadolu University, Eskişehir TR-26470, Turkey (e-mail: ongerek@anadolu.edu.tr).

A. E. Çetin is with the Department of Electrical and Electronics Engineering, Bilkent University, Bilkent, Ankara TR-06533, Turkey.

Digital Object Identifier 10.1109/TIP.2005.859369

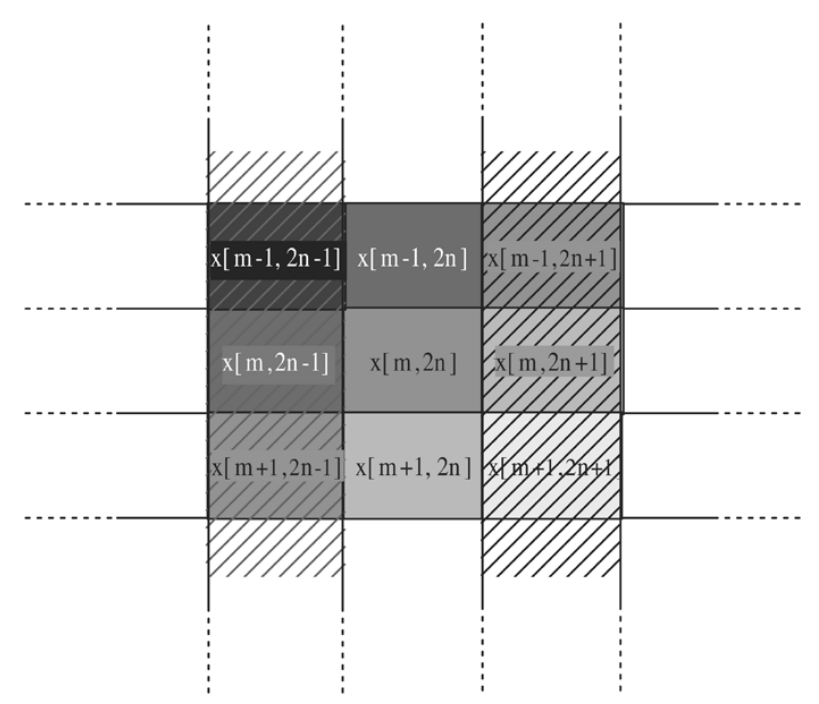

Fig. 1. Sample image segment.

sequently, for a one-dimensional (1-D) input signal $x[n], y_{0}[n]$ and $y_{1}[n]$ correspond to the approximation and detail signals generated at the output of the decomposition. In order to distinguish between the directional delay elements in two-dimensional (2-D) processing, we use $z_{h}^{-1}$ and $z_{v}^{-1}$ as the horizontal and vertical delay elements.

\section{INTRODUCTION}

The 5/3 Daubechies biorthogonal wavelet has received a wide range of interest in various applications due to its rational filter tap coefficients which are particularly useful in real-time implementations. Furthermore, the lifting implementation of this wavelet contains filters with coefficients that can be written as powers of two leading to a multiplication free realization of the filter bank [1], [2]. Several linear or nonlinear decomposition structures that are published in the literature report better performance than the $5 / 3$ wavelet using signal adapted filters, including [3]-[7]. In [2], it has been shown that any DWT filter bank can be decomposed into series of lifting/dual-lifting steps. The work of [3] extends the idea of linear filters in the lifting style to nonlinear filters. In [4], [8], and [7], the lifting prediction filter was made adaptive according to the local signal properties, and in [6], the importance of coder-nonlinear transform strategy was emphasized. The idea of lifting adaptation was also applied to video processing [9], [10]. Finally, in [5], [11], and [12], 2-D extensions of the lifting structures were examined, which fundamentally resembles the idea of this work. Due to the fact that the 5/3 wavelet system has filter coefficients that are dyadic rationals, a very fast, efficient and integer-shift-only implementa- 
tion is possible. As a consequence, it was a natural choice in the JPEG-2000 lossless image coding standard [13], [14].

The subband filter coefficients of the $5 / 3$ wavelet are: $h_{0}=[-1 / 8,1 / 4,3 / 4,1 / 4,-1 / 8]$ and $h_{1}=[-1 / 2,1,-1 / 2]$. Its lifting implementation is very efficient and can be realized using binary shifting operations due to coefficients with dyadic rationals of 2 as follows:

$$
\begin{aligned}
y_{1}[n]= & x[2 n]-\frac{1}{2}(x[2 n-1]+x[2 n+1]) \\
y_{0}[n]= & x[2 n-1]+\frac{1}{4}\left(y_{1}[n-1]+y_{1}[n]\right) \\
= & -\frac{1}{8} x[2 n-3]+\frac{1}{4} x[2 n-2] \\
& +\frac{3}{4} x[2 n-1]+\frac{1}{4} x[2 n]-\frac{1}{8} x[2 n+1] .
\end{aligned}
$$

Notice that prediction filter is very short, consisting of an averaging operation performed over the left and right neighboring samples in a row (or column) in 2-D image processing. The lifting structure corresponding to (1) is shown in Fig. 2.

The above lifting implementation is purely 1-D. In other words, the image is processed line by line during implementation. In 2-D separable extension of the above filterbank the image is first processed horizontally (or vertically) and then processed vertically (or horizontally) to obtain four subband images. Let us consider horizontal processing of the image around a pixel $x[m, 2 n]$. The prediction filter inherently assumes that the right and left neighbor pixels are closely related with the pixel between them. As a result, $(x[m, 2 n-1]+x[m, 2 n+1]) / 2$ will be an accurate estimate of $x[m, 2 n]$. Hence, by subtracting this prediction value from the true value of $x[m, 2 n]$, a small residue is obtained. This residual signal corresponds to the detail signal obtained after the single stage $5 / 3$ wavelet transformation. If there is an edge close to the center pixel, $x[m, 2 n]$ may be unrelated to $x[m, 2 n-1]$ or $x[m, 2 n+1]$. On the other hand, some of the four other immediate diagonal neighbors $x[m+1,2 n+1], x[m+1,2 n-1], x[m-1,2 n+1]$ and $x[m-1,2 n-1]$ may be closer to the pixel $x[m, 2 n]$ in value. Therefore, it may be better to use two of these four diagonal neighbors in the prediction stage of the lifting structure in a judicious manner. Our adaptive predictor is obtained by relaxing the condition that the predictor should use samples from the current row that is being processed. In Section III, a computationally efficient adaptation strategy describing how to switch from single-line horizontal processing to multiline horizontal processing is presented. The predictor can still use only two pixels for computational efficiency as in [1]; however, it can select them from the neighboring rows instead of the current row. Resultant lifting scheme can still be implemented in two stages consisting of a row-wise processing followed by a column-wise processing as in ordinary lifting.

The proposed analysis filterbank does neither require any multiplications nor transmission of any side information during implementation. Due to its locally adaptive nature, this work may be categorized in a class of works reported in [8]-[12]. It was also reported in [11] that such multiline lifting realizations can be performed in a memory-efficient manner.

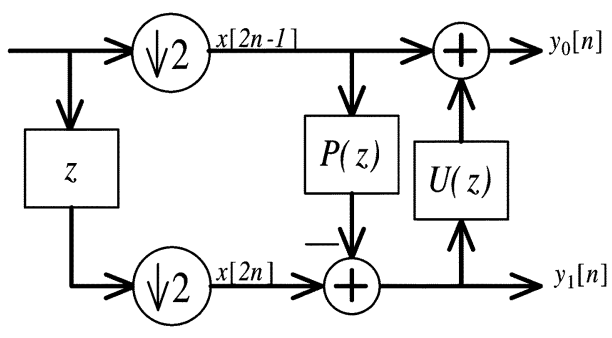

Fig. 2. Lifting analysis stage.

\section{Edge Sensitive AdAPtive Prediction}

The algorithm described in this paper was inspired from a work describing CCD imaging systems and missing pixel value interpolation in color filter arrays (CFAs) [15]. The CFA interpolator in [15] estimates the missing pixel $x[m, 2 n]$ using its immediate 4-neighbors according to the following principles.

- If $\Delta_{180}<T$ and $\Delta_{0}>T, T$ being a specified threshold, then the vertical pixel transition is slow and horizontal pixel transition is sharp, so the interpolation value is $\hat{x}_{180}[m, 2 n]$.

- Else, if $\Delta_{180}>T$ and $\Delta_{0}<T$, then the horizontal pixel transition is slow and vertical pixel transition is sharp, so the interpolation value is $\hat{x}_{0}[m, 2 n]$.

- Else, pixel transitions are similar in both directions, so the interpolation value is $\left(\hat{x}_{0}[m, 2 n]+\hat{x}_{180}[m, 2 n]\right) / 2$.

This rule gives a good approximation of a possibly missing color sensor output, so it improves both the mean-square error and the subjective quality of the image after reconstruction. Since the scales are only $1 / 2$ or $1 / 4$, the interpolator can be implemented efficiently using bitwise shift operations.

In this paper, a similar interpolation scheme is developed in the prediction part of a lifting stage in image processing. The predictor does not have to be limited to use samples from the same row (or column in columnwise processing). Let us assume horizontal processing, without any loss of generality. In ordinary lifting implementation, the available polyphase samples for prediction of pixel $x[m, 2 n]$ must belong to polyphase component 1, which are shown by dashed pixels in Fig. 1. Therefore, $\hat{x}_{180}[m, 2 n]$ is not available as a prediction value for $x[m, 2 n]$. On the other hand, since polyphase component 1 is completely available for prediction, $\hat{x}_{135}[\mathrm{~m}, 2 n]$ and $\hat{x}_{45}[m, 2 n]$ can be safely used as well as the 1-D predictor $\hat{x}_{0}[m, 2 n]$. The ordinary $5 / 3$ biorthogonal wavelet lifting structure uses $\hat{x}_{0}[m, 2 n]=1 / 2(x[m, 2 n-1]+x[m, 2 n+1])$ for predicting the value of $x[m, 2 n]$. We introduce four more diagonal neighboring samples from the upper and lower rows, hence, enable the use of $\hat{x}_{135}[m, 2 n]$ and $\hat{x}_{45}[m, 2 n]$ as possible prediction values. In fact, if the local gradient is in the south-east direction (as illustrated in Fig. 1), then there is more possibility that the center of the $3 \times 3$ region has a pixel value similar to its diagonal neighbors $(x[m+1,2 n-1]$ and $x[m-1,2 n+1]$, which are in a direction orthogonal to the local gradient. This concept is generalized to the other directions according to the following adaptation rule for the selection of prediction domain pixels.

- If $\Delta_{135}$ is the least among $\Delta_{135}, \Delta_{0}$, and $\Delta_{45}$, then the prediction estimate is $\hat{x}_{135}[\mathrm{~m}, 2 n]$. 
- If $\Delta_{0}$ is the least among $\Delta_{135}, \Delta_{0}$, and $\Delta_{45}$, then the prediction estimate is $\hat{x}_{0}[m, 2 n]$.

- If $\Delta_{45}$ is the least among $\Delta_{135}, \Delta_{0}$, and $\Delta_{45}$, then the prediction estimate is $\hat{x}_{45}[m, 2 n]$.

In the example shown in Fig. 1, the largest gradient is in the south-east direction. As a result, $\Delta_{45}$ is the minimum difference. Therefore, the value of $x[m, 2 n]$ must be predicted as $\hat{x}_{45}[m, 2 n]$. It must be noted that such a tilted prediction $(P(z))$ does not require transmission of any side information, because the pixels used in prediction and the pixel to be predicted belong to different poly-phase components. In case of no quantization, these columns are automatically reconstructed and the decoder uses the same directional choice method that was used in encoder.

The resultant 2-D analysis filters can be constructed as follows. In 1-D single-line processing, the subsignals $y_{0}[n]$ and $y_{1}[n]$, are related to even $x_{e}[n]$ and odd $x_{o}[n]$ components of the signal $x[n]$ via the relation

$$
\left[\begin{array}{c}
Y_{0}(z) \\
Y_{1}(z)
\end{array}\right]=\left[\begin{array}{cc}
1-P(z) U(z) & U(z) \\
-P(z) & 1
\end{array}\right]\left[\begin{array}{l}
X_{e}(z) \\
X_{o}(z)
\end{array}\right]
$$

If the lifting implementation is carried out using ordinary singleline processing, the polyphase transform matrix takes the following form in the $z$ domain:

$$
\left[\begin{array}{cc}
1-\frac{1}{8}(1+z)\left(1+z^{-1}\right) & \frac{1}{4}\left(1+z^{-1}\right) \\
-\frac{1}{2}(1+z) & 1
\end{array}\right] .
$$

This matrix provides the coefficient information to generate the analysis filters in a filter-bank structure

$$
\left[\begin{array}{ll}
H_{0, \mathrm{ev}}(z) & H_{0, \mathrm{odd}}(z) \\
H_{1, \mathrm{ev}}(z) & H_{1, \mathrm{odd}}(z)
\end{array}\right]
$$

and $H_{i}(z)=H_{i, \mathrm{ev}}\left(z^{2}\right)+z^{-1} H_{i, \mathrm{odd}}\left(z^{2}\right)$, for $i=0$, 1 . Considering a 2-D signal, if multiline processing is performed, the delay elements $z_{v}^{-1}$ and $z_{h}^{-1}$ must be used simultaneously. For example, for the $45^{\circ}$ prediction direction, the polyphase matrix becomes

$$
\left[\begin{array}{cc}
1-\frac{1}{8}\left(z_{v}^{-1}+z_{v} \cdot z_{h}\right)\left(1+z_{h}^{-1}\right) & \frac{1}{4}\left(1+z_{h}^{-1}\right) \\
-\frac{1}{2}\left(z_{v}^{-1}+z_{v} \cdot z_{h}\right) & 1
\end{array}\right] .
$$

The low-pass and high-pass filters of the filter-bank corresponding to the matrix in (5) are directional 2-D filters in the spatial domain.

Considering the horizontal process of the image, a drawback of the above adaptive lifting structure is that the approximation coefficient $y_{0}[m, n]$ is generated from polyphase samples $(x[m, 2 n])$ that are predicted from other rows' polyphase samples $(x[m+1,2 n-1]$ and $x[m-1,2 n+1])$. Therefore, there is a row-wise lifting update leakage. Because of this leakage, the effect of $U(z)$ in the lifting stage deviates from anti-aliasing low-pass filter. This situation leads to distortions in low-low subimages across decomposition scales. This problem can be solved by changing the order of the update $U(z)$ and the prediction $P(z)$ stages of Fig. 2 as discussed in Section IV. With the proper choice of the low-pass filter, the new $U(z)$ can be performed prior to the prediction, and its implementation still requires no multiplications, so the computational efficiency is retained.

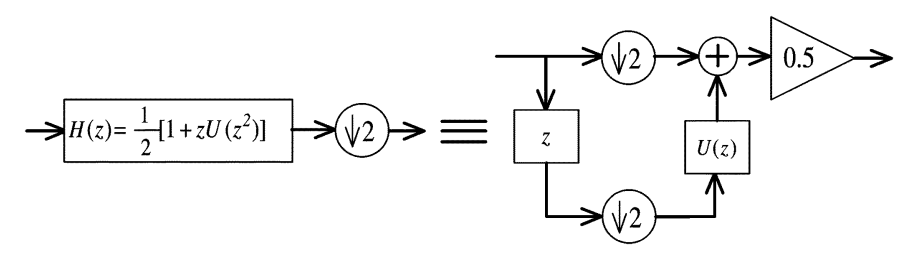

Fig. 3. Lifting update implementation of a half-band filter.

\section{AdAPtive Lifting Structure Performing LOW-PASS FILTERING FIRST}

High-quality, low-low images can be obtained by performing low-pass filtering first in a lifting structure. A half-band lowpass filter can be put into an isolated update lifting stage as in [4].

In order to achieve a multiplierless structure we consider the simple length-3 Lagrangian half-band low-pass filter: $\mathbf{h}_{l_{3}}=$ $\{1 / 4,1 / 2,1 / 4\}$. The $z$ transform of this filter is

$$
H_{l_{3}}(z)=\frac{1}{2}\left(1+z\left(U\left(z^{2}\right)\right)\right.
$$

where $U(z)=(1 / 2) z^{-1}+(1 / 2)$. This low-pass filter followed by down sampling can be implemented in a lifting structure due to the relation known as Noble-Identity. The resulting structure is shown in Fig. 3. Since $U(z)$ is a very simple update filter consisting of dyadic rationals of 2 , it can be implemented using bit-wise shift operations.

After this stage, the adaptive prediction algorithm described above can be applied. Since the low-pass filtering is performed first, the low-low subimages are as good as those obtained by any subband decomposition structure using the third-order Lagrange half-band filter.

The overall structure including the low-pass filter is still computationally comparable to the original implementation of the Daubechies 5/3 wavelet in terms of calculations per lifting operation.

\section{EXPERIMENTAL RESULTS AND CONCLUSION}

The selection of prediction domain in the lifting stage has a number of practical advantages. We have experimentally observed that, in a typical test image, among $\hat{x}_{135}[m, 2 n]$, $\hat{x}_{0}[m, 2 n]$, and $\hat{x}_{45}[m, 2 n]$, the possibility of the horizontal process $\left(\hat{x}_{0}[m, 2 n]=1 / 2(x[m, 2 n-1]+x[m, 2 n+1])\right)$ being the best prediction of $x[m, 2 n]$ is $30.1 \%$. This is slightly less than about one-thirds of the possible predictions. As a result, persistently using horizontal prediction loses chances of making better prediction decisions. On the other hand, our directionally sensitive prediction decision rule catches about $52 \%$ of the best predictions as described above. This improvement reflects to practical compression results, too.

In the absence of quantization, perfect reconstruction of the proposed algorithm is assured due to the symmetric lifting implementations in encoding and decoding stages. On the other hand, the choice of minimum $\Delta_{135}, \Delta_{0}$, and $\Delta_{45}$ has a chance to alter if the transform coefficients are quantized. However, it is experimentally observed that this does not occur with wavelet tree bitplane coders at compression ratios down to 0.5 bpp for 


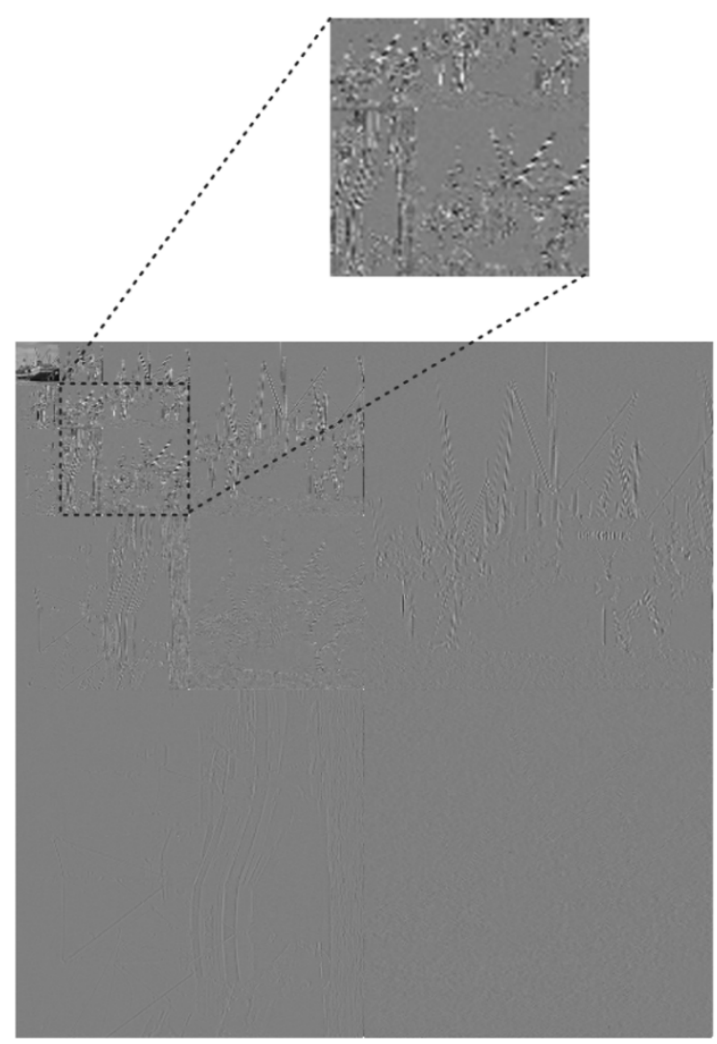

(a)

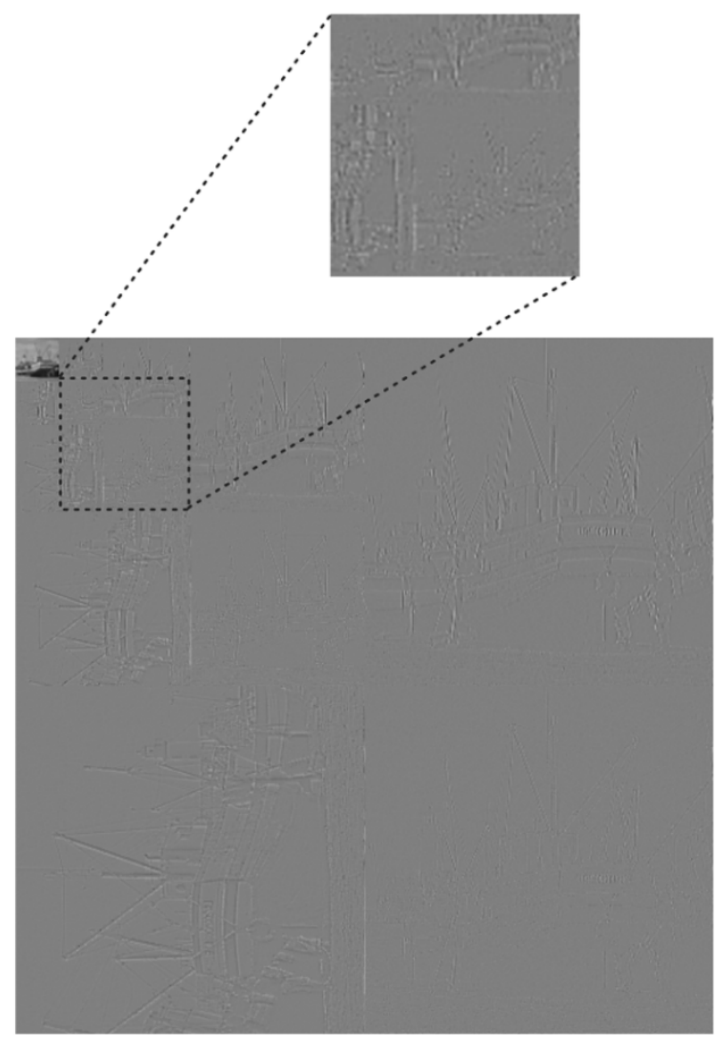

(b)

Fig. 4. Wavelet trees obtained by (a) regular 5/3 wavelet and (b) our method.

TABLE I

EXPERIMENTAL RESUltS FOR $512 \times 512$ TeSt IMAges (SAMPLE VARIANCE $=\sigma^{2}$ AND SAMPLE ENTROPY $=I$ ) AT 1 bpp AND 0.5 bpp

\begin{tabular}{l|c|c|c|c}
\hline & $\begin{array}{c}\text { Orig. 5/3 } \\
\text { at 1bpp }\end{array}$ & $\begin{array}{c}\text { Our method } \\
\text { at 1bpp }\end{array}$ & $\begin{array}{c}\text { Orig. 5/3 } \\
\text { at 0.5bpp }\end{array}$ & $\begin{array}{c}\text { Our method } \\
\text { at 0.5bpp }\end{array}$ \\
\hline boats $\left(\sigma^{2}=2466, \mathrm{I}=7.03\right)$ & 37.04 & 37.20 & 33.30 & 33.33 \\
\hline airfield $\left(\sigma^{2}=3594, \mathrm{I}=7.12\right)$ & 28.48 & 29.01 & 26.70 & 26.81 \\
\hline bridge $\left(\sigma^{2}=2996, \mathrm{I}=5.71\right)$ & 27.93 & 28.00 & 25.61 & 25.59 \\
\hline harbor $\left(\sigma^{2}=1565, \mathrm{I}=6.76\right)$ & 32.40 & 32.58 & 28.14 & 28.20 \\
\hline lena $\left(\sigma^{2}=2290, \mathrm{I}=7.44\right)$ & 37.11 & 37.10 & 33.75 & 33.69 \\
\hline barbara $\left(\sigma^{2}=2982, \mathrm{I}=7.63\right)$ & 32.70 & 32.68 & 28.21 & 28.13 \\
\hline houses $\left(\sigma^{2}=2423, \mathrm{I}=7.48\right)$ & 33.48 & 33.61 & 31.14 & 31.19 \\
\hline garden $\left(\sigma^{2}=5665, \mathrm{I}=7.52\right)$ & 31.47 & 31.69 & 24.22 & 24.42 \\
\hline topkapi $\left(\sigma^{2}=6314, \mathrm{I}=7.28\right)$ & 34.24 & 34.35 & 29.29 & 29.35 \\
\hline duomo $\left(\sigma^{2}=1256, \mathrm{I}=6.92\right)$ & 28.08 & 28.21 & 24.85 & 24.93 \\
\hline peppers $\left(\sigma^{2}=2894, \mathrm{I}=7.59\right)$ & 35.65 & 35.71 & 33.20 & 33.21 \\
\hline
\end{tabular}

8-bpp originals. Below this bit rate, the orientation selection rule of the decoder starts to deviate from that of the encoder at arbitrary image locations. This shows that the direction adaptation rule is fairly robust to fine quantization, and the proposed method is suitable for relatively high bit rate compression.

In Fig. 4, (a) original 5/3 wavelet decomposition and (b) directionally modified prediction lifting decomposition images of one of the test images are shown, respectively. Notice that the detail images obtained by the directionally adaptive $5 / 3$ wavelet exhibits less signal energy at several decomposition levels in general. In this example, the high-pass coefficients in Fig. 4(a) have a variance $=94.06$ and a sample entropy $=3.4536$, whereas the high-pass coefficients in Fig. 4(b) have variance $=30.57$ and sample entropy $=3.4412$. This behavior is observed in other test images, as well. The energy reduction shows that better compression results can be obtained using our method, as compared to the $5 / 3$ wavelet in high-band subimages.

For presenting practical results, a bitplane compression method [13], [14] is used to encode the transform domain coefficients. A decomposition level of 4 was selected for $512 \times 512$ images. The PSNR values for a set of test images at 1 and $0.5 \mathrm{bpp}$ are shown in Table I for our directionally adaptive method using the half-band anti-aliasing update filter and the Daubechies 5/3 wavelet. The same encoder is used in both cases. In general, slightly better PSNRs are obtained for a given compression level in our filterbank.

The proposed method better preserves sharp edges of the original image compared to the ordinary $5 / 3$ wavelet decomposition. This is because of the reduced high-band signal 


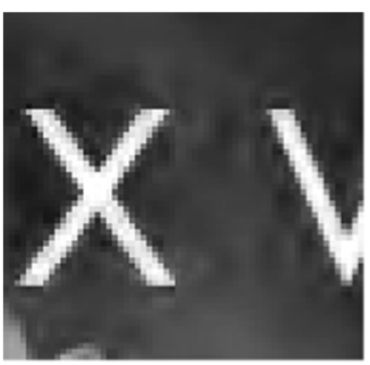

(a)

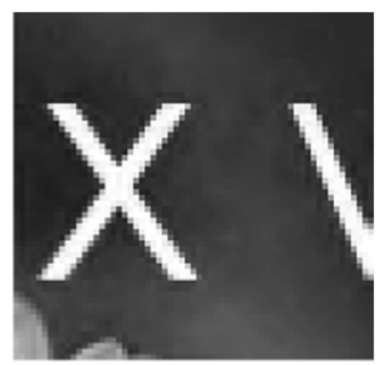

(b)
Fig. 5. A detail from "garden" image coded at 0.5 bpp using (a) the $5 / 3$ wavelet and (b) our method.

energy at diagonal edge locations compared to the $5 / 3$ wavelet decomposition. The following example illustrates the visual improvement obtained by our method. The test image is listed as "garden" in Table I and it contains printed text on a natural flower background. Small portions of images from $0.5 \mathrm{bpp}$ coded versions of this image are shown in Fig. 5. Fig. 5(a) shows the regular 5/3 wavelet coded version, and Fig. 5(b) shows the result from our method. Edges are sharper with less ringing artifacts in Fig. 5(b) due to better prediction around diagonal edges of the original image.

In spite of the edge adaptation of the prediction, the overall proposed method gives marginally better or similar PSNR values as compared to the $5 / 3$ wavelet. The reason for this situation is due to the low-pass filtering prior to the prediction. This filter automatically reduces some amount of prediction information in the upper polyphase component. We have observed that a combination of the given low-pass filter followed by a 1-D prediction filter (as used in the $5 / 3$ wavelet) gives worse PSNR results than the original 5/3 wavelet. By incorporating the 2-D orientation adaptations, the PSNR results improve to better than or comparable with the $5 / 3$ wavelet. On the other hand, the use of the low-pass filter in the upper polyphase is essential because without this filter, the downsampling process yields aliased low-low images which are harder to process in the later decomposition stages.

The computational complexity of the proposed adaptive filter-bank is very low. Our directionally adaptive lifting strategy contains an additional

1) three difference operations to obtain $\Delta_{135}, \Delta_{0}$, and $\Delta_{45}$;

2) three comparison operations to choose the minimum of $\Delta_{135}, \Delta_{0}$, and $\Delta_{45}$.

This is in comparison to Daubechies 5/3 wavelet decomposition.

The rest of the operations, including the anti-aliasing filtering, have identical complexity figures as the original $5 / 3$ lifting implementation. The above operations can be summarized as an additional complexity of six subtractions per lifting (including prediction and update) operation. For an $N \times N$ image, there are approximately $N^{2}$ lifting operations, so the additional computational cost is $6 N^{2}$ subtractions. There is neither any integer nor floating point multiplications in the new structure. As a result, our directionally adaptive algorithm keeps the low complexity property of the 5/3 Daubechies wavelet decomposition, and provides slightly better image compression results in images containing sharp edges and artificial characters and drawings around $1 \mathrm{bpp}$.

\section{ACKNOWLEDGMENT}

The authors would like to thank the reviewers for their valuble comments during the review process of this paper.

\section{REFERENCES}

[1] W. Sweldens, "The lifting scheme: A new philosophy in biorthogonal wavelet constructions," in Proc. SPIE Wavelet Applications in Signal and Image Processing III, vol. 2569, A. F. Laine and M. Unser, Eds., 1995, pp. 68-79.

[2] I. Daubechies and W. Sweldens, "Factoring wavelet transforms into lifting steps," J. Fourier Anal. Appl., vol. 4, no. 3, pp. 247-269, 1998.

[3] R. L. Claypoole et al., "Nonlinear wavelet transforms for image coding via lifting," IEEE Trans. Image Process., vol. 12, no. 12, pp. 1449-1459, Dec. 2003.

[4] O. N. Gerek and A. E. Çetin, "Adaptive polyphase subband decomposition structures for image compression," IEEE Trans. Image Process., vol. 9, no. 10, pp. 1649-1660, Oct. 2000.

[5] A. Gouze, M. Antonini, M. Barlaud, and B. Mack, "Design of signaladapted multidimensional lifting scheme for lossy coding," IEEE Trans. Image Process., vol. 13, no. 12, pp. 1589-1603, Dec. 2004

[6] A. Cohen, I. Daubechies, O. G. Guleryuz, and M. T. Orchard, "On the importance of combining wavelet-based nonlinear approximation with coding strategies," IEEE Trans. Inf. Theory, vol. 48, no. 7, pp. 1895-1921, Jul. 2002.

[7] T. Chan and H. M. Zhou, "Adaptive ENO-Wavelet Transforms for Discontinuous Functions,” UCLA Rep. no. CAM 99-21, 1999.

[8] G. Piella, B. Pesquet-Popescu, and H. Heijmans, "Adaptive update lifting with a decision rule based on derivative filters," IEEE Signal Process. Lett., vol. 9, no. 10, pp. 329-332, Oct. 2002.

[9] G. Pau, C. Tillier, and B. Pesquet-Popescu, "Optimization of the predict operator in lifting-based motion compensated temporal filtering," presented at the SPIE VCIP, San Jose, CA, Jan. 2004.

[10] N. Mehrseresht and D. Taubman, "Adaptively weighted update steps in motion compensated lifting based on scalable video compression," in Proc. IEEE Int. Conf. Image Processing, vol. 2, Sep. 2003, pp. 771-774.

[11] D. Taubman, "Adaptive, nonseparable lifting transforms for image compression," in Proc. IEEE Int. Conf. Image Processing, vol. 3, Oct. 1999, pp. 772-776.

[12] H. Heijmans, G. Piella, and B. Pesquet-Popescu, "Building adaptive 2-D wavelet decompositions by update lifting," presented at the IEEE Int. Conf. Image Processing, Rochester, NY, Oct. 2002.

[13] JPEG-2000 Part-1 Standard, ISO/IEC 15 444-1.

[14] C. Christopoulos, A. Skodras, and T. Ebrahimi, "The JPEG2000 still image coding system: An overview," IEEE Trans. Consum. Electron., vol. 46, no. 6, pp. 1103-1127, Nov. 2000.

[15] R. H. Hibbard, "Apparatus and Method for Adaptively Interpolating a Full Color Image Utilizing Luminance Gradients," U.S. Patent 5382976 .

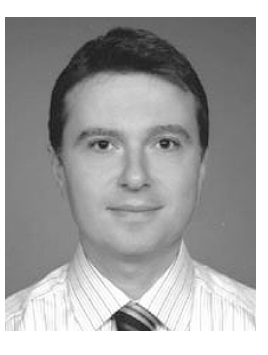

Ömer N. Gerek (S'89-M'98) was born in Eskisehir, Turkey, in 1969. He received the B.Sc., M.Sc., and Ph.D. degrees in electrical engineering from Bilkent University, Ankara, Turkey, in 1991, 1993, and 1998, respectively.

Following the Ph.D. degree, he spent one year as a Research Associate at EPFL, Lausanne, Switzerland. Currently, he is a Professor of electrical engineering at Anadolu University, Eskisehir. His research areas include signal analysis, image coding, wavelets, and subband decomposition. 


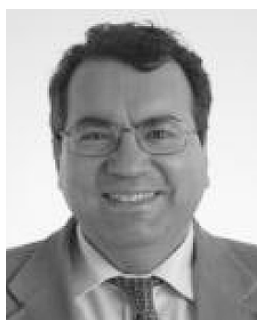

A. Enis Çetin (S'85-M'87-M'95) received the B.S. degree in electrical engineering from the Middle East Technical University, Ankara, Turkey, and the M.S.E and $\mathrm{Ph} . \mathrm{D}$. degrees in systems engineering from the Moore School of Electrical Engineering, University of Pennsylvania, Philadelphia.

From 1987 to 1989, he was an Assistant Professor of electrical engineering at the University of Toronto, Toronto, ON, Canada. Since then, he has been with Bilkent University, Ankara. Currently, he is a Full Professor. During the summers of 1988, 1991 and 1992, he was with Bell Communications Research (Bellcore) as a Consultant. He spent the 1996 and 1997 academic years at the University of Minnesota, Minneapolis, as a Visiting Professor. He carried out contract research for both governmental agencies and industry, including Visioprime, U.K.; Honeywell Video Systems, Grandeye, U.K.; the National Science Foundation; NSERC, Canada; and ASELSAN.

Prof. Çetin is a senior member EURASIP. Currently, he is a scientific committee member of the EU FP6 funded Network of Excellence (NoE) MUSCLE: Multimedia Understanding through Semantics, Computation and Learning. From 1999 to 2003, he was an Associate Editor of the IEEE TRANSACTIONS ON IMAGE PROCESSING, which is the most prestigious journal in the image processing area. He is a member of the DSP technical committee of the IEEE Circuits and Systems Society. He founded the Turkish Chapter of the IEEE Signal Processing Society in 1991. He was Signal Processing and AES Chapter Coordinator of IEEE Region-8 in 2003. He was the Co-Chair of the IEEE-EURASIP Nonlinear Signal and Image Processing Workshop, held in 1999 in Antalya, Turkey, and the technical Co-Chair of the European Signal Processing Conference (EUSIPCO) in 2005. He received the Young Scientist Award from the Turkish Scientific and Technical Research Council (TUBITAK) in 1993. 